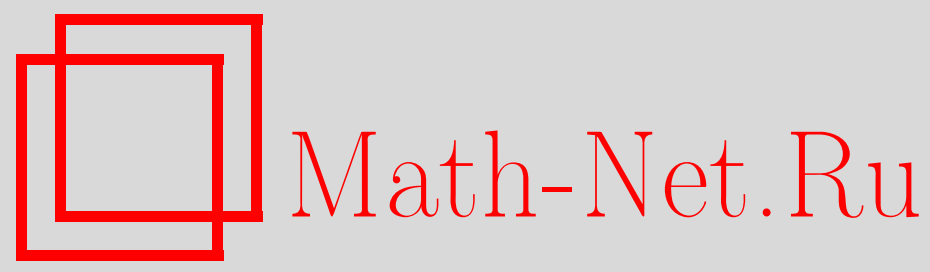

Л. П. Власов, Чебышевские подпространства конечной коразмерности в комплексном $C(Q)$, Матем. заметки, 1997, том 62, выпуск 2, 178-191

DOI: https://doi.org/10.4213/mzm1603

Использование Общероссийского математического портала Math-Net.Ru подразумевает, что вы прочитали и согласны с пользовательским соглашением http://www.mathnet.ru/rus/agreement

Параметры загрузки:

IP : 44.207 .124 .84

26 апреля 2023 г., 15:39:44

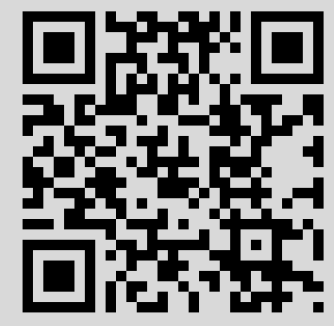




\section{ЧЕБЫШЕВСКИЕ ПОДПРОСТРАНСТВА КОНЕЧНОЙ КОРАЗМЕРНОСТИ В КОМПЛЕКСНОМ $C(Q)$}

\section{Л.П. Власов}

Рассматриваются чебьшевские подпространства конечной коразмерности в комплексном $C(Q)$, где $Q$ - бикомпакт. Доказываются аналоги теорем, установленных ранее для вещественного случая А. Л. Гаркави и А. Брауном, например, дается характеризация таких подпространств. Показано, что если в вещественном $C(Q)$ существуют чебышевские подпространства конечной коразмерности, то они существуют и в комплексном $C(Q)$ (для того же $Q$ ).

Библиограффия: 8 названий.

Статья примыкает к исследованиям [1]-[4] и непосредственно продолжает работу автора [5].

Множество $M$ в нормированном пространстве $X$ называется проксиминальным, или множеством существования, если для любого $x \in X$ множество элементов наилучшего приближения $P_{M} x=\{y \in M:\|x-y\|=d(x, M)\}$ непусто. Если $P_{M} x$ одноточечно, то $M$ назьвается чебышевским. В дальнейшем $C(Q)$ - комплексное (вещественное) пространство непрерьвных на бикомпакте $Q$ комплексных (вешественных) функций с обычной нормой; $C(Q)^{*}$ - пространство мер Радона на $Q,\|\mu\|=|\mu| Q=\operatorname{var}(\mu, Q)$; $S_{\mu}=\{t \in Q:|\mu| G>0$ для любого открытого $G \ni t\} ; Q^{\prime}(I)$ - множество предельных (изолированных) точек бикомпакта $Q$ (предполагается, что $Q$ бесконечно); $\Sigma$ $\sigma$-алгебра борелевских подмножеств $Q ; e, E$ (также с индексами) обозначают всегда множества из $\Sigma ; \partial A$ - граница, $\stackrel{\circ}{A}$ - внутренность, $|A|$ - мошность множества $A ; \nu \ll \mu$ на $A$ означает, что мера $\nu$ абсолютно непрерьвна относительно $\mu$ на множестве $A$ (если $A=Q$, пишется просто $\nu \ll \mu)$. Нам понадобится следующая

ТЕОрема 1. Для того чтобы подпространство L конечной коразмерности в комплексном или вещественном $C(Q)$ было множсеством существования необходимо и достаточно, чтобы выполнялись условия:

(1а) для любой меры $\mu \in L^{\perp}$ существует непрерывная производная Радона-Никодима $d \mu / d|\mu|$

(1б) для любых $\mu, \nu \in L^{\perp}$ множсество $S_{\nu} \backslash S_{\mu}$ замкнуто;

(1в) для любых $\mu, \nu \in L^{\perp}$ мера $\nu$ абсолютно непрерывна относительно $\mu$ на мнохестве $S_{\mu}$.

Работа выполнена при финансовой поддержке Российского фонда фундаментальных исследований, грант № 93-011-196. 
В вещественном случае теорема установлена Гаркави [2], в комплексном - автором [5].

Свойства производной Радона-Никодима оформим в виде леммы (см. [6, гл. III, п. 10]).

Лемма 1. 1) Ecлu $d \mu / d \nu=f$, m.e. $\mu e=\int_{e} f(t) d \nu \quad \forall e \in \Sigma$, mo $|\mu| e=\int_{e}|f(t)| d|\nu|$ $u d \nu / d \mu=1 / f$ на $\left\{t \in S_{\nu}: f(t) \neq 0\right\}$. Ecли, кроме того, $d \nu / d \lambda=\varphi$, то $d \mu / d \lambda=d \mu / d \nu \cdot d \nu / d \lambda=f \varphi$, m.e. $\mu e=\int_{e} f d \nu=\int_{e} f \varphi d \lambda$.

2) $E c л и f=d \mu / d|\mu|$, то $|f(t)|=1$ н-почти всюду.

3) Если $g \in C(Q),\|g\| \leqslant 1$, то $|\mu| Q=\int_{Q} g(t) d \mu$ тогда и только тогда, когда $g=d|\mu| / d \mu$.

Утверждение 3) леммы см. в [5], [7].

Лемма 2. Пусть $\mu, \nu \in C(Q)^{*}$. Тогда существует такое счетное $R_{\mu \nu} \subset \mathbb{C}$, что для всех $z \in \mathbb{C} \backslash R_{\mu \nu}$ меры $\mu$ и $\nu$ абсолютно непрерывны относительно меры $\mu+z \nu$ u $S_{\mu+z \nu}=S_{\mu} \cup S_{\nu}$. Следовательно, для $L \subset C(Q), \operatorname{codim} L<\infty$, существует $\nu \in L^{\perp} c \lambda \ll \nu \quad \forall \lambda \in L^{\perp}$,

$$
S_{\nu}=\bigcup_{\lambda \in L^{\perp}} S_{\lambda} .
$$

Аналогичное утверждение для вешественного случая доказано в [8, лемма 10].

ДоКАЗАТЕЛЬСТВО. Положим

$$
R_{\mu \nu}=\left\{z \in \mathbb{C}: \exists e_{z} \in \Sigma \mathrm{c}|\mu| e_{z}+|\nu| e_{z}>0,|\mu+z \nu| e_{z}=0\right\}
$$

Для $z, z^{\prime} \in R_{\mu \nu}, z \neq z^{\prime}, e \subset e_{z} \cap e_{z^{\prime}}$ имеем

$$
|\mu+z \nu| e=\left|\mu+z^{\prime} \nu\right| e=0, \quad \mu e+z \nu e=\mu e+z^{\prime} \nu e=0, \quad \nu e=0, \quad \mu e=0 .
$$

Так как $e \subset e_{z} \cap e_{z^{\prime}}$ произвольно, то $|\mu|\left(e_{z} \cap e_{z^{\prime}}\right)=|\nu|\left(e_{z} \cap e_{z^{\prime}}\right)=0$. Отсюда $\left\{z \in R_{\mu \nu}\right.$ : $\left.|\mu| e_{z}+|\nu| e_{z}>1 / n\right\}$ конечно, а $R_{\mu \nu}$ не более чем счетно. Если $\mu_{1}, \ldots, \mu_{n}-$ базис в $L^{\perp}$, то, последовательно применяя доказанное утверждение к мерам $\sum_{i=1}^{k} z_{i} \mu_{i}$ и $\mu_{k+1}$, получим

$$
Q=\bigcup_{1}^{n} S_{\mu_{i}}=S_{\nu}
$$

где $z_{1}=1, k=1, \ldots, n-1, \nu=\sum_{1}^{n} z_{i} \mu_{i} \in L^{\perp}$.

ТЕОрема 2. Для того чтоби подпространство $L$ коразмерности $n$ в комплексном или вещественном $C(Q)$ было чебышевским множеством необходимо $u$ достаточно, чтобь выполнялись условия:

(2а) для любой меры $\mu \in L^{\perp}$ существует непрерывная производная Радона-Никодима $d \mu / d|\mu|$

(2б) $\left|Q \backslash S_{\mu}\right|<n \quad \forall \mu \in L^{\perp} \backslash\{0\}$;

(2в) любые меры $\mu, \nu \in L^{\perp} \backslash\{0\}$ взаимно абсолютно непрерывны на $Q^{\prime}$;

$(2 г)$ если $\left\{\mu_{1}, \mu_{2}, \ldots, \mu_{n}\right\}-$ базис в $L^{\perp}, I=Q \backslash Q^{\prime}=\left\{t_{1}, t_{2}, \ldots, t_{q}\right\} u q<n$, mo $\operatorname{rank}\left\|\mu_{i}\left\{t_{k}\right\}\right\|_{k \leqslant q}^{i \leqslant n}=q$.

В вещественном случае аналогичная теорема установлена Гаркави [2] (другие варианты см. также в [4]). 
ДокАЗАТЕЛЬСтво. Необходимость. Условие (2а) выполняется в силу теоремы 1. Докажем (2б) и (2г). Предположим противное. Тогда или

(А) $\left|Q \backslash S_{\mu}\right| \geqslant n$ для некоторой $\mu \in L^{\perp} \backslash\{0\}$, или

(Б) $|I|<n$, Ho $\operatorname{rank}\left\|\lambda_{i}\left\{t_{k}\right\}\right\|<|I|$.

Найдем $r$ различных точек $t_{1}, t_{2}, \ldots, t_{r}$ из $Q \backslash S_{\mu}$ в $(\mathrm{A})$ или из $I$ в (Б), при этом $r=n$ в (А) и $r=q$ в (Б). В случае (Б) ввиду $r<n$ тоже имеется мера из $L^{\perp} \backslash\{0\}$ (обозначим ее, как и в (А), через $\mu$ ) с $t_{k} \in Q \backslash S_{\mu} \forall k \leqslant r$. Пусть $G_{k}$ - открытые множества такие, что $\bar{G}_{k} \subset Q \backslash S_{\mu}, t_{k} \in G_{k}, \bar{G}_{k} \cap \bar{G}_{j}=\varnothing(k \neq j)$; вешественные функции $g_{k} \in C(Q)$ такие, что $g_{k}\left(t_{k}\right)=1, g_{k}(t)=0 \forall t \notin G_{k},\left\|g_{k}\right\|=1$ (в случае (Б) $G_{k}=\left\{t_{k}\right\}, g_{k}=\chi_{\left\{t_{k}\right\}}$ ). Рассмотрим базис $\mu_{1}, \mu_{2}, \ldots, \mu_{n}$ в $L^{\perp}$, где $\mu_{1}=\mu$. Система $n-1$ уравнений с $r$ неизвестными

$$
\sum_{k=1}^{r} \alpha_{k} a_{i k}=0, \quad i=2,3, \ldots, n
$$

где $a_{i k}=\int_{G_{k}} g_{k} d \mu_{i}\left(a_{i k}=\mu_{i}\left\{t_{k}\right\}\right.$ в случае $($ Б $\left.)\right)$, имеет ненулевое решение $\left(\alpha_{1}, \ldots, \alpha_{r}\right)$, ибо $r=n$ в $(\mathrm{A}), \operatorname{rank}\left\|a_{i k}\right\|<r=q$ в (Б). Возьмем

$$
g=\sum_{k=1}^{r} \alpha_{k} g_{k}
$$

тогда $g \in L \backslash\{0\}$. Можно считать $\|\mu\|=1$. Положим $x=d|\mu| / d \mu$ на $S_{\mu}, x=0$ на $F=\bigcup_{1}^{r} \bar{G}_{k}$ и продолжим до $x \in C(Q)$ с $\|x\|=1\left(d|\mu| / d \mu\right.$ непрерывна на $S_{\mu}$ по теореме 1 и лемме 1). Покажем, что $\varepsilon g \in P_{L} x$ для всех достаточно малых $\varepsilon \geqslant 0$ :

$$
\begin{aligned}
\|x-\varepsilon g\| & =\max \left\{\max _{t \in Q \backslash F}|x(t)-\varepsilon g(t)|, \max _{k} \max _{t \in G_{k}}\left|x(t)-\varepsilon \alpha_{k} g_{k}(t)\right|\right\} \\
& \leqslant \max \left\{\|x\|, \max _{k}\left|\varepsilon \alpha_{k}\right|\right\}=\|x\|,
\end{aligned}
$$

если $\varepsilon\left|\alpha_{k}\right|<\|x\|$. Итак, $\varepsilon g \in P_{L} x$, что противоречит единственности.

$(2 \mathrm{~B})$ вьполняется по теореме 1 на множестве $S_{\mu} \cap S_{\nu}=Q \backslash\left[\left(Q \backslash S_{\mu}\right) \cup\left(Q \backslash S_{\nu}\right)\right]$, которое содержит множество $Q^{\prime}$ в силу (2б).

Достаточность. $L$ проксиминально по теореме 1: (1a) совпадает с (2a); (1б) следует из $(2 б)$ ввиду $S_{\nu} \backslash S_{\mu}=S_{\nu} \cap\left(Q \backslash S_{\mu}\right) ;(1 \mathrm{~B})$ - из (2в), так как из $е \subset S_{\mu},|\mu| e=0$ следует, что $e \subset Q^{\prime}$.

Допустим, что $L$ не чебышевское, тогда найдутся $x \notin L, \mu \in L^{\perp} \backslash\{0\}$ и $g \in L \backslash\{0\}$ такие, что

$$
\|x\|=\|x-g\|=d(x, L)=\int_{Q} x d \mu=\int_{Q}(x-g) d \mu=\|\mu\|=1
$$

(см., например, [7]). Из этих равенств следует (см. лемму 1), что $x\left|S_{\mu}=(x-g)\right| S_{\mu}=$ $d|\mu| / d \mu$, поэтому $g=0$ на $S_{\mu}$. Далее, если $Q \backslash S_{\mu}=\left\{t_{1}, \ldots, t_{r}\right\}$, то

$$
g=\sum_{1}^{r} \alpha_{k} \chi_{\left\{t_{k}\right\}}, \quad \alpha_{k} \in \mathbb{C} .
$$

Заметим теперь (см. [2]), что

(В) если $|I| \geqslant n$, то для различньх $t_{1}, \ldots, t_{n} \in I \operatorname{det}\left\|\mu_{i}\left\{t_{k}\right\}\right\| \neq 0$ 
(иначе некоторая мера из $L^{\perp}$ обратилась бы в 0 навсех $t_{k}$ вопреки $(2 б)$ ). Если же $|I|<n$, то в силу $(2 г)$

$$
\operatorname{rank}\left\|\mu_{i}\left\{t_{k}\right\}\right\|_{k=1, \ldots, q}^{i=1, \ldots, n}=q .
$$

Но ввиду $g \in L \backslash\{0\}$

$$
\sum_{k=1}^{r} \alpha_{k} \mu_{i}\left\{t_{k}\right\}=0, \quad i=1, \ldots, n,
$$

т.е. столбцы матрицы $\left\|\mu_{i}\left\{t_{k}\right\}\right\|$ линейно зависимы, что противоречит сказанному ранее, ибо $r \leqslant \min \{q, n-1\}$.

СлЕДСТВИЕ 1. Если L чебышевское, то

$$
Q=\bigcup_{\lambda \in L^{\perp}} S_{\lambda}
$$

и существует $\nu \in L^{\perp}$ c $S_{\nu}=Q$.

ДокАЗАтЕльство. Из (2б) следует, что $Q \backslash S_{\lambda} \subset I \forall \lambda \in L^{\perp} \backslash\{0\}$, а из (В) и (2г) вытекает, что случай $\lambda\{t\}=0 \forall \lambda \in L^{\perp}$ невозможен для $t \in I$. Остается сослаться на лемму 2.

СЛЕДСТВИЕ 2. Для того чтобы подпространство

$$
L=\{\nu\}_{\perp}=\left\{x \in C(Q): \int_{Q} x d \nu=0\right\}
$$

коразмерности 1 в комплексном или вещественном $C(Q)$ было чебышевским множеством необходимо и достаточно, чтобы выполнялись условие (2а) и равенство $S_{\nu}=Q$.

ЗАмЕчАниЕ 1. Следствие 2 нельзя считать новым даже в комплексном случае (см., например, [7]).

Далее мы будем обозначать через $\widetilde{\mathbb{C}}$ комплексную плоскость, дополненную "бесконечно удаленньми точками" вида $\infty \cdot w$, где $w \in \partial D$, т.е. $w \in \mathbb{C},|w|=1(D=\{z \in \mathbb{C}$ : $|z| \leqslant 1\})$. Предполагается, что $z_{n} \rightarrow \infty \cdot w$, если $\left|z_{n}\right| \rightarrow \infty, z_{n} /\left|z_{n}\right| \rightarrow w ; \infty \cdot w_{n} \rightarrow \infty \cdot w$ при $w_{n} \rightarrow w,(\infty \cdot w) \cdot\left(\infty \cdot w^{\prime}\right)=\infty \cdot w w^{\prime},(\infty \cdot w) \cdot 0=0, \infty \cdot 0=0$. Топологически $\widetilde{\mathbb{C}}$ эквивалентна “диску" $D$.

В последуюшем мы пишем (ср. [4]) $\mu e / \nu e \rightarrow z \in \mathbb{C}$ при $e \rightarrow t \in Q$, если для любого $\varepsilon>0$ найдется окрестность $G$ точки $t$ такая, что $|\mu e / \nu e-z|<\varepsilon$, как только $e-$ борелевское подмножество $G, \nu e \neq 0$ (предполагается, что $t \in S_{\nu}$ ). Для "бесконечно удаленной точки" $\mu е / \nu e \rightarrow \infty \cdot w$, если

$$
\left|\frac{\mu e}{\nu e}\right| \rightarrow \infty, \quad \frac{\mu e / \nu e}{|\mu e / \nu e|} \rightarrow w
$$

ЛЕмма 3. Если $d \mu / d|\mu|=h$ непрерывна в точке $t_{0} \in S_{\mu}$, mо $\mu e /|\mu| e \rightarrow h\left(t_{0}\right)$, $\mu e /|\mu e| \rightarrow h\left(t_{0}\right),|\mu e| /|\mu| e \rightarrow 1$ при $е \rightarrow t_{0}$. Ecли $d \mu / d \nu=f u d \nu / d|\nu|=g$ непрерывнь

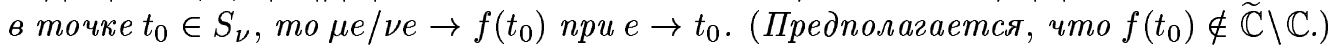


ДокАЗАТЕЛЬСТво. Используя определение производной Радона-Никодима, имеем

$$
\left|h\left(t_{0}\right)-\frac{\mu e}{|\mu| e}\right|=\left|\int_{e}\left[h\left(t_{0}\right)-h(t)\right] d\right| \mu|| /|\mu| e<\varepsilon \quad \forall e \subset G,
$$

если $G \ni t_{0}$ выбрано из условия $\left|h\left(t_{0}\right)-h(t)\right|<\varepsilon \forall t \in G$. Далее,

$$
\frac{|\mu e|}{|\mu| e} \rightarrow 1, \quad \frac{\mu e}{|\mu e|}=\frac{\mu e /|\mu| e}{|\mu e /| \mu|e|} \rightarrow \frac{h\left(t_{0}\right)}{\left|h\left(t_{0}\right)\right|}=h\left(t_{0}\right),
$$

так как по лемме $1|h(t)|=1 \mu$-почти всюду, следовательно, во всех точках непрерьвности. Наконец, если $d \nu / d|\nu|=g$ непрерывна в точке $t_{0}$, то $|\nu e| /|\nu| e \rightarrow 1$ по доказанному, и

$$
\left|f\left(t_{0}\right)-\frac{\mu e}{\nu e}\right|=\left|\int_{e}\left[f\left(t_{0}\right)-f(t)\right] d \nu\right| /|\nu e| \leqslant \varepsilon \frac{|\nu| e}{|\nu e|} \leqslant 2 \varepsilon,
$$

если $G \ni t_{0}$ выбрано из условий $\left|f\left(t_{0}\right)-f(t)\right|<\varepsilon \forall t \in G$ и $|\nu| e \leqslant 2|\nu e| \forall e \subset G$.

Лемма 4. Пусть подпространство L удовлетворяет условию (1a), $\mu, \nu \in L^{\perp}$, $t_{0} \in S_{\mu} \cap S_{\nu}$. Тогда существуют $\theta \in[0, \infty] u w \in \mathbb{C},|w|=1$, такие, ито при $е \rightarrow t_{0}$ $(\mu e \neq 0, \nu e \neq 0)$

$$
\frac{\mu e}{\nu e} \rightarrow \theta \cdot w, \quad\left|\frac{\mu e}{\nu e}\right| \rightarrow \theta, \quad \frac{\mu e / \nu e}{|\mu e / \nu e|} \rightarrow w .
$$

ДоКАЗАТЕЛЬСТво. ПоЛожим

$$
w_{e}=\frac{\mu e / \nu e}{|\mu e / \nu e|}
$$

тогда

$$
w_{e}=\frac{\mu e /|\mu e|}{\nu e /|\nu e|}
$$

и по (1a) и лемме 3 найдутся $w, w_{0} \in \partial D, w_{e} \rightarrow w, \nu e /|\nu e| \rightarrow w_{0}$. Покажем, что $|\mu e / \nu e|$ сходится (возможно, к $\infty$ ). В противном случае существуют направленности $e_{\alpha}, e_{\beta}^{\prime} \rightarrow t_{0},\left|\mu e_{\alpha} / \nu e_{\alpha}\right| \rightarrow \theta,\left|\mu e_{\beta}^{\prime} / \nu e_{\beta}^{\prime}\right| \rightarrow \theta^{\prime}, 0 \leqslant \theta<\theta^{\prime} \leqslant \infty$. Для $z=\theta_{1} w$, где $\theta<\theta_{1}<\theta^{\prime}$, и $\lambda=\mu-z \nu \in L^{\perp}$ имеем $\lambda e=\nu e\left(w_{e}|\mu e / \nu e|-z\right)$ и

$$
\frac{\lambda e_{\alpha}}{\left|\lambda e_{\alpha}\right|}=\frac{\nu e_{\alpha}}{\left|\nu e_{\alpha}\right|} \cdot \frac{w_{e_{\alpha}}\left|\mu e_{\alpha} / \nu e_{\alpha}\right|-z}{\left|w_{e_{\alpha}}\right| \mu e_{\alpha} / \nu e_{\alpha}|-z|} \rightarrow w_{0} \frac{w \theta-z}{|w \theta-z|}, \quad \frac{\lambda e_{\beta}^{\prime}}{\left|\lambda e_{\beta}^{\prime}\right|} \rightarrow w_{0} \frac{w \theta^{\prime}-z}{\left|w \theta^{\prime}-z\right|} .
$$

Эти пределы различны при $z=\theta_{1} w$. При этом по лемме 2 можно считать $z \notin R_{\mu \nu}$, тогда $t_{0} \in S_{\mu} \cup S_{\nu}=S_{\lambda}$ вопреки (1а) и лемме 3.

Используя лемму 4 , определим три функции

$$
\theta: S_{\nu} \rightarrow[0, \infty], \quad w: S_{\nu} \cap S_{\mu} \rightarrow \partial D, \quad f: S_{\nu} \rightarrow \widetilde{\mathbb{C}}
$$

такие, что при $e \rightarrow t \in S_{\nu} \cap S_{\mu}$

$$
\left|\frac{\mu e}{\nu e}\right| \rightarrow \theta(t), \quad \frac{\mu e / \nu e}{|\mu e / \nu e|} \rightarrow w(t), \quad \frac{\mu e}{\nu e} \rightarrow f(t)=\theta(t) \cdot w(t),
$$

а если $t \in S_{\nu} \backslash S_{\mu}$, то полагаем $f(t)=\theta(t)=0$. 
ЛЕмма 5. При условиях леммы 4 функиии $\theta, w, f$ непрерьвны.

ДоказАтельство. Пусть вначале $\theta\left(t_{0}\right)<\infty, t_{0} \in S_{\nu}$. Будем считать $t_{0} \in S_{\mu}$, так как случай $t_{0} \in S_{\nu} \backslash S_{\mu}$ ясен из определения $\theta$. Для любого $\varepsilon \in(0,1 / 2)$ найдем по лемме 4 открытое $G \ni t_{0} \mathrm{c}|\mu e / \nu e| \in\left(\theta\left(t_{0}\right)-\varepsilon, \theta\left(t_{0}\right)+\varepsilon\right) \forall e \subset G$. Возьмем $t \in G$ и найдем открытое $G_{1} \ni t, G_{1} \subset G$ с $|\mu e / \nu e| \in(\theta(t)-\varepsilon, \theta(t)+\varepsilon) \forall e \subset G_{1}$ (в случае $t \in S_{\mu}$ по лемме 4 , а случай $t \in S_{\nu} \backslash S_{\mu}$ очевиден). Так как при этом $e \subset G$, то ясно, что $\left|\theta(t)-\theta\left(t_{0}\right)\right|<2 \varepsilon$ в случае $\mu e \neq 0$. Если же $\mu e=0$, то возьмем $e^{\prime} \in G$ такое, что $0<\left|\mu e^{\prime}\right|<\varepsilon|\nu e|,\left|\nu e^{\prime}\right|<\varepsilon|\nu e|$, и положим $E=e \cup e^{\prime}$. Имеем

$$
E \subset G, \quad \mu E \neq 0, \quad\left|\frac{\mu E}{\nu E}\right|=\frac{\left|\mu e^{\prime}\right|}{\left|\nu e+\nu e^{\prime}\right|} \leqslant \frac{\varepsilon|\nu e|}{|\nu e|-\left|\nu e^{\prime}\right|}<2 \varepsilon .
$$

Тогда $\left|\theta(t)-\theta\left(t_{0}\right)\right|<3 \varepsilon$. Аналогично доказываются случай $\theta\left(t_{0}\right)=\infty$ и непрерывность $w(t), f(t)$ с использованием соглашений относительно $\infty$ и $\widetilde{\mathbb{C}}$.

В дальнейшем будем считать $w$ продолженной на $S_{\nu}$ до непрерьвной функции со значениями в $D$. (Как известно, при таком продолжении не всегда можно сохранить равенство $w(t)=1$.) Обозначим

$$
S_{\nu}^{\prime}=\left\{t \in S_{\nu}: \theta(t)<\infty\right\}=\left\{t \in S_{\nu}: f(t) \in \widetilde{\mathbb{C}} \backslash \mathbb{C}\right\} .
$$

Лемма 6. Пусть подпространство L удовлетворяет условию (1a), для мер $\mu, \nu \in L^{\perp}$ определены, как в лемме 4 , функиии $\theta, w, f$. Тогда $|\nu|\left(S_{\nu} \backslash S_{\nu}^{\prime}\right)=0, a$

$$
\theta=\frac{d|\mu|}{d|\nu|}, \quad f=\frac{d \mu}{d \nu} \quad \text { намножестве } S_{\nu}^{\prime} .
$$

Если дополнительно L удовлетворяет условию (1в), то (1) выполняется на $S_{\nu}$.

ДоказАтельство. Обозначим $e=S_{\nu} \backslash S_{\nu}^{\prime}$. Тогда $e \subset S_{\mu}$ и замкнуто по непрерьвности $\theta$ ( $S_{\nu}^{\prime}$ открыто в $\left.S_{\nu}\right)$. Для произвольного $\varepsilon>0$ и каждого $t \in e$ найдется открытое $G_{t} \ni t \mathrm{c}|\nu| e^{\prime} \leqslant \varepsilon|\mu| e^{\prime} \forall e^{\prime} \subset G_{t}$ по определению $\theta(t)$. Пусть $\left\{G_{t_{i}}\right\}$ - конечное покрытие $e$. Сушествуют $e_{i} \subset G_{t_{i}}$ с $e_{i} \cap e_{j}=\varnothing(i \neq j), e=\bigcup_{i} e_{i}$. Тогда $|\nu| e=\sum|\nu| e_{i} \leqslant \varepsilon \sum|\mu| e_{i}=\varepsilon|\mu| e \leqslant \varepsilon\|\mu\|$, следовательно, $|\nu|\left(S_{\nu} \backslash S_{\nu}^{\prime}\right)=0$.

Покажем теперь, что для $e \subset S_{\nu}^{\prime}$

$$
|\mu| e=\int_{e} \theta(t) d|\nu|
$$

Пусть вначале $e \subset S_{\nu}^{n}=\left\{t \in S_{\nu}: \theta(t) \leqslant n\right\}$. Для каждого $t \in S_{\nu}^{n}$ по леммам 4,5 найдется открытое $G_{t} \ni t$ такое, что

$$
\left|\theta(t)-\frac{|\mu| E}{|\nu| E}\right|<\varepsilon \quad \forall E \subset G_{t}
$$

c $|\nu| E \neq 0,\left|\theta(t)-\theta\left(t^{\prime}\right)\right|<\varepsilon \forall t^{\prime} \in G_{t}$. Пусть $\left\{G_{t_{i}}\right\}$ - конечное покрытие $S_{\nu}^{n}$. Тогда

$$
e=\bigcup_{i} e_{i}, \quad e_{i} \subset G_{t_{i}}, \quad e_{i} \cap e_{j}=\varnothing \quad(i \neq j), \quad|| \mu\left|e_{i}-\theta\left(t_{i}\right)\right| \nu\left|e_{i}\right| \leqslant \varepsilon|\nu| e_{i}
$$


(легко видеть, что $\mu \ll \nu$ на множестве $G_{t}$ ). Имеем

$$
\begin{aligned}
|| \mu\left|e-\int_{e} \theta(t) d\right| \nu|| & =\left|\sum\left\{|\mu| e_{i}-\int_{e_{i}} \theta(t)|\nu|(d t)\right\}\right| \\
& =\left|\sum\left\{\left[|\mu| e_{i}-\theta\left(t_{i}\right)|\nu| e_{i}\right]+\int_{e_{i}}\left[\theta\left(t_{i}\right)-\theta(t)\right]|\nu|(d t)\right\}\right| \\
& \leqslant 2 \varepsilon \sum|\nu| e_{i}=2 \varepsilon|\nu| e .
\end{aligned}
$$

Это и означает, что

$$
|\mu| e=\int_{e} \theta(t) d|\nu|
$$

т.е. $\theta=d|\mu| / d|\nu|$ на $S_{\nu}^{n}$. Положим теперь $\theta_{n}(t)=\theta(t)$, если $\theta(t) \leqslant n$, и $\theta_{n}(t)=0$, если $\theta(t)>n\left(t \in S_{\nu}\right)$. Пусть $\theta_{n} \nu$-измерима на $S_{\nu}, 0 \leqslant \theta_{n} \leqslant \theta_{n+1}, \theta_{n} \rightarrow \theta$ на $S_{\nu}^{\prime}$. Тогда, как известно [5, III.6.17],

$$
\int_{S_{\nu}^{\prime}} \theta(t) d|\nu|=\lim _{n \rightarrow \infty} \int \theta_{n}(t) d|\nu|=\lim _{n \rightarrow \infty} \int_{S_{\nu}^{n}} \theta(t) d|\nu|=\lim _{n \rightarrow \infty}|\mu| S_{\nu}^{n} \leqslant\|\mu\|,
$$

т.е. $\theta \nu$-интегрируема на $S_{\nu}^{\prime}$. Если теперь $e \subset S_{\nu}^{\prime}$, то

$$
e=\bigcup_{n=1}^{\infty} e_{n}
$$

где

$$
e_{n} \subset S_{\nu}^{n}, \quad e_{m} \cap e_{n}=\varnothing, \quad|\mu| e=\sum|\mu| e_{n}=\sum \int_{e_{n}} \theta(t) d|\nu|=\int_{e} \theta(t) d|\nu| .
$$

Таким образом, $\theta=d|\mu| / d|\nu|$ на $S_{\nu}^{\prime}$, а ввиду $|\nu|\left(S_{\nu} \backslash S_{\nu}^{\prime}\right)=0$ и на $S_{\nu}$ по соглашению $\infty \cdot 0=0$ и при условии $(1 \mathrm{~B})$, ибо тогда и $|\mu|\left(S_{\nu} \backslash S_{\nu}^{\prime}\right)=0$.

Осталось показать, что $\theta \cdot w=d \mu / d \nu$ на $S_{\nu}^{\prime}$. Поскольку

$$
w(t)=\lim _{e \rightarrow t} \frac{\mu e / \nu e}{|\mu e / \nu e|}=\lim _{e \rightarrow t} \frac{\mu e /|\mu e|}{\nu e /|\nu e|}=\frac{h(t)}{g(t)},
$$

то по лемме 1

$$
\int_{e} \theta \cdot w d \nu=\int_{e} \theta \cdot w \cdot g d|\nu|=\int_{e} w g d|\mu|=\int_{e} \frac{w g}{h} d \mu=\mu e
$$

где $e \subset S_{\nu}^{\prime}, h=d \mu / d|\mu|, g=d \nu / d|\nu|$. При условии (1в) равенство $\mu e=\int_{e} \theta(t) w(t) d \nu$ распространяется на $e \subset S_{\nu}$ так же, как равенство $|\mu| e=\int_{e} \theta(t) d|\nu|$ вьше.

Обозначим

$$
N_{z}=N_{z, f}=\left\{t \in S_{\nu}: f(t)=z\right\}, \quad \text { где } z \in \mathbb{C}, \quad f \in C\left(S_{\nu}, \widetilde{\mathbb{C}}\right) .
$$


Лемма 7. Пусть $\mu, \nu \in C(Q)^{*}, z \in \mathbb{C}, \lambda=\mu-z \nu u L=\{\mu, \nu\}_{\perp}$ удовлетворяет условию (1a). Тогда

$$
S_{\lambda}=\left(S_{\mu} \backslash S_{\nu}\right) \cup \overline{S_{\nu} \backslash N_{z, f}},
$$

əде $f=d \mu / d \nu \in C\left(S_{\nu}, \widetilde{\mathbb{C}}\right)$, а если выполняется (1в), то

$$
S_{\lambda}=\left(S_{\mu} \backslash S_{\nu}\right) \cup \overline{S_{\nu}^{\prime} \backslash N_{z, f}}
$$

ДокаЗАТЕЛЬСтво. Заметим, что $\lambda e=\mu e \forall e \subset S_{\mu} \backslash S_{\nu}$, так что $S_{\lambda} \backslash S_{\nu}=S_{\mu} \backslash S_{\nu}$. Пусть $t^{\prime} \in S_{\nu} \backslash N_{z}, G \ni t^{\prime}$ открыто и $G \cap N_{z}=\varnothing$. Тогда, используя лемму 1 , из $\lambda e=\int_{e}(f(t)-z) d \nu \forall e \in \Sigma$ получим, что

$$
|\lambda| G=\int_{G}|f(t)-z| d|\nu|>0, \quad t^{\prime} \in S_{\lambda}, \quad S_{\nu} \backslash N_{z} \subset S_{\lambda}, \quad\left(S_{\mu} \backslash S_{\nu}\right) \cup \overline{S_{\nu} \backslash N_{z}} \subset S_{\lambda} .
$$

Пусть, далее, $t^{\prime} \in S_{\lambda} \backslash\left(S_{\mu} \backslash S_{\nu}\right)$, но $t^{\prime} \notin \overline{S_{\nu}^{\prime} \backslash N_{z}}$. Тогда найдется открытое $G \ni t^{\prime}$, $G \cap\left(S_{\nu}^{\prime} \backslash N_{z}\right)=\varnothing$, т.е. $G \cap S_{\nu}^{\prime} \subset N_{z}$, и по лемме 6

$$
|\lambda| G=\int_{G \cap S_{\nu}^{\prime}}|f(t)-z| d|\nu|+\int_{G \cap\left(S_{\nu} \backslash S_{\nu}^{\prime}\right)}|f(t)-z| d|\nu|=0
$$

что невозможно из-за $t^{\prime} \in S_{\lambda}$. Если вместо $S_{\nu}^{\prime}$ в этих рассуждениях подставить $S_{\nu}$, то получим (2), при этом не используется (1в). Лемма доказана.

Лемма 8. Пусть $\mu, \nu \in C(Q)^{*}, z \in \mathbb{C}, \lambda=\mu-z \nu, f=d \mu / d \nu, g=d \nu / d|\nu|$. Тогда

$$
\frac{d \lambda}{d|\lambda|}(t)=\frac{f(t)-z}{|f(t)-z|} g(t) \quad \lambda \text {-почти всюдуна } S_{\nu} \backslash N_{z, f} .
$$

ДокАЗАТЕЛЬСТво. Принимая во внимание лемму 1 , имеем

$$
\lambda e=\int_{e}(f(t)-z) d \nu=\int_{e}(f(t)-z) g(t) d|\nu|=\int_{e} \frac{f(t)-z}{|f(t)-z|} g(t) d|\lambda|
$$

где $e \subset S_{\nu} \backslash N_{z}$, что и означает (3).

ЗАМЕчАние 2 . В случае $f(t)=\infty \cdot w$ естественно считать, что

$$
\psi_{z}(t)=\frac{f(t)-z}{|f(t)-z|}=w
$$

причем $\psi_{z}$ непрерывна на $S_{\nu} \backslash N_{z}$, если $f \in C\left(S_{\nu}, \widetilde{\mathbb{C}}\right)$. 
Теорема 3. Пусть $\mu, \nu \in C(Q)^{*}, S_{\nu}$ открыто-замкнуто в $Q$. Для того чтобь подпространство

$$
L=\{\mu, \nu\}_{\perp}=\left\{x \in C(Q): \int_{Q} x d \mu=\int_{Q} x d \nu=0\right\}
$$

было множеством существования достаточно, а если $\mu, \nu$ линейно независимы, то и необходимо, чтобы выполнялись условия:

(3a) cyществует $g=d \nu / d|\nu| \in C(Q)$;

(3б) существует $f=d \mu / d \nu \in C\left(S_{\nu}, \widetilde{\mathbb{C}}\right)$;

(3в) $\overline{Q \backslash N_{z, f}}$ открыто (или, равносильно, $\stackrel{\circ}{N}_{z, f}$ замкнуто) $\forall z \in \mathbb{C}$;

(3г) $|\nu|\left(\partial N_{z, f}\right)=0 \quad \forall z \in \mathbb{C}$;

(3д) $\forall z \in \mathbb{C}$ в каждой точке $t_{0} \in \partial N_{z, f}$ существует предел

$$
\lim _{\substack{t \rightarrow t_{0} \\ t \in Q \backslash N_{z, f}}} \frac{f(t)-z}{|f(t)-z|}
$$

(т.е. существует продолжсение $\psi_{z} \in C(Q)$ функиии $(f(t)-z) /|f(t)-z|$ на $Q)$.

ДокаЗАТЕЛЬСтво. Необходимость. (3а) следует из (1а), (3б) - из лемм 5 и 6.

(3в) Возьмем $\lambda=\mu-z \nu, z \in \mathbb{C}$. Тогда по лемме $7 S_{\lambda}=\left(S_{\mu} \backslash S_{\nu}\right) \cup \overline{S_{\nu} \backslash N_{z}}$, $\overline{S_{\nu} \backslash N_{z}}=S_{\nu} \cap S_{\lambda}=S_{\nu} \backslash\left(S_{\nu} \backslash S_{\lambda}\right)$ открыто в силу (1б). Так как $Q \backslash S_{\nu}$ открыто-замкнуто по условию, то $Q \backslash \stackrel{\circ}{N}_{z}=\overline{Q \backslash N_{z}}=\overline{Q \backslash S_{\nu}} \cup \overline{S_{\nu} \backslash N_{z}}$ открыто.

(Зг) Ясно, что $|\lambda| N_{z}=0$ для $z \in \mathbb{C}$, а так как согласно (1в) $\nu \ll \lambda$ на $S_{\lambda} \supset \partial N_{z}$, то $|\nu|\left(\partial N_{z}\right)=0$.

(3д) Для $\lambda=\mu-z \nu$ выполняется (3). В силу (1а) можно положить

$$
\psi_{z}=\frac{1}{g} \frac{d \lambda}{d|\lambda|}
$$

ибо $1 / g \in C(Q)$ по доказанному (3а) и лемме 1 .

Достаточность. Проверим условия теоремы 1. Возьмем произвольное $\lambda \in L^{\perp} \backslash\{0\}$. Имеем $\lambda=\alpha \mu+\beta \nu, \alpha, \beta \in \mathbb{C}$.

(1а) Если $\alpha=0$, то $d \lambda / d|\lambda|=d \nu / d|\nu|$ непрерьвна по условию (3а). При $\alpha \neq 0$ можно считать, что $\alpha=1, \lambda=\mu-z \nu$, где $z=-\beta \in \mathbb{C}$. Очевидно, ввиду (3) и (3д) можно положить $d \lambda / d|\lambda|=\psi_{z} g \in C(Q)$.

(1б) Так как (1а) доказано, по лемме 7 имеем (2), тогда $S_{\lambda}=\left(S_{\mu} \cup S_{\nu}\right) \backslash \stackrel{\circ}{N_{z}}$ открыто относительно $S_{\mu} \cup S_{\nu}$ согласно (Зв) (случай $\alpha=0$ очевиден), а это и означает (1б).

(1в) Ввиду (3б) $\mu \ll \nu$ на $S_{\nu}$, и значит, $\lambda \ll \nu$ на $S_{\nu} \forall \lambda \in L^{\perp}$. Докажем, что $\nu \ll \lambda$ на $S_{\lambda}$ (случай $\alpha=0$ опять очевиден). Пусть $\lambda=\mu-z \nu$. По лемме $1 d \nu / d \lambda=1 /(f(t)-z)$, $\nu \ll \lambda$ на $S_{\nu} \backslash N_{z}$. Так как $S_{\lambda}=\left(S_{\mu} \backslash S_{\nu}\right) \cup\left(S_{\nu} \backslash N_{z}\right) \cup \partial N_{z}$ по лемме 7 и доказанному (1а), то в силу (3г) $\nu \ll \lambda$ на $S_{\lambda} \forall \lambda \in L^{\perp}$. Отсюда следует (1в). 
ЗАмЕчАниЕ 3 . Отказ от условия “ $S_{\nu}$ открыто-замкнуто в $Q$ ” возможен, но приводит к излишней сложности формулировок: топологические понятия - открытость, внутренность, граница - придется снабдить указанием “относительно $S_{\nu}$ ”. Действительно, лемма 2 показьвает, что можно найти $\nu \in L^{\perp}$ с

$$
S_{\nu}=\bigcup_{\lambda \in L^{\perp}} S_{\lambda}
$$

а по теореме 1 проксиминальность $L$ равносильна проксиминальности подпространства

$$
L^{\prime}=\left\{x \in C\left(Q^{\prime \prime}\right): \int_{Q^{\prime \prime}} x d \mu=\int_{Q^{\prime \prime}} x d \nu=0\right\},
$$

где бикомпакт $Q^{\prime \prime}$ равен $S_{\nu}$. То же самое можно сказать относительно следующей теоремы.

ТЕОРема 4. Пусть $L$ - подпространство конечной коразмерности в комплексном или вещественном $C(Q)$,

$$
\bigcup_{\lambda \in L^{\perp}} S_{\lambda}=Q .
$$

Для того чтобы L било мнохсеством существования необходимо и достаточно, чтобы для некоторой (любой) меры $\nu \in L^{\perp}$ такой, что $\lambda \ll \nu$ для всех $\lambda \in L^{\perp}$ (см. лемму 2), и любой меры $\mu \in L^{\perp}$, линейно независимой $c \nu$, выполнялись условия (3а)-(3д).

ДокАЗАТЕЛЬСтво. Необходимость. Подпространство $\{\mu, \nu\}_{\perp}-$ множество сушествования (по теореме 1 ) - имеет коразмерность 2 и $S_{\nu}=Q$. По теореме 3 вьполняются условия (3a)-(3д).

Достаточность. Пусть для некоторой $\nu$ и любой $\mu$, указанных в теореме, вьполняются условия (3а)-(3д). Проверим условия теоремы 1.

(1a) Если $\mu$ линейно зависит от $\nu$, то (1a) выполняется ввиду (3a). В силу (3a), (3б), (3д) при $z=0$ и леммы $1 d \mu / d|\mu|=f g /|f| \in C(Q)$.

(1б) Ввиду (2) и (3в) $S_{\mu}=\overline{Q \backslash N_{0}}$ открыто, а $S_{\lambda} \backslash S_{\mu}$ замкнуто $\forall \mu, \lambda \in L^{\perp}$.

(1в) Пусть $\lambda, \mu \in L^{\perp}$ произвольны и $\nu \in L^{\perp}$ удовлетворяет указанным в теореме условиям. Тогда $\lambda \ll \nu$, а так как $L^{\prime}=\{\mu, \nu\}_{\perp}-$ множество существования по теореме 3 , то $\nu \ll \mu$ на $S_{\mu}$ по теореме 1 , примененной к $L^{\prime}$. Поэтому и $\lambda \ll \mu$ на $S_{\mu}$.

Теорема 5. Пусть $\mu, \nu \in C(Q)^{*}$ линейно независимы. Для того чтобы подпространство $L=\{\mu, \nu\}_{\perp}$ в комплексном или вещественном $C(Q)$ было чебышевским мнохеством необходимо и достаточно, чтобы выполнялись условия:

(5а) существуют производные Радона-Никодима

$$
g=\frac{d \nu}{d|\nu|} \in C(Q), \quad f=\frac{d \mu}{d \nu} \in C\left(S_{\nu}, \widetilde{\mathbb{C}}\right) ;
$$

(5б) $S_{\mu} \cup S_{\nu}=Q u\left|S_{\mu} \backslash S_{\nu}\right| \leqslant 1$;

(5в) $\left|\stackrel{\circ}{N}_{z, f}\right| \leqslant 1 \quad \forall z \in \mathbb{C}$;

(5г) $|\nu|\left(\partial N_{z, f}\right)=0 \quad \forall z \in \mathbb{C}$

$(5$ д) для любого $z \in \mathbb{C}$ функиия $(f(t)-z) /|f(t)-z|$, определенная в точках $t \in S_{\nu} \backslash N_{z, f}$, продолжается до функиии $\psi_{z} \in C(Q)$. 
ДокаЗАТЕЛЬСтво. Необходимость. Используем теорему 2 при $n=2$. (5б) вьполняется ввиду следствия 1 и (2б). Так как $\stackrel{\circ}{N}_{z} \subset Q \backslash S_{\lambda}$, то (5в) вытекает из (2б). Поскольку $S_{\nu}$ открыто-замкнуто (его дополнение конечно), то по теореме 3 вьполняются условия (5а), (5г), (5д).

Достаточность. Проверим условия теоремы 2 при $n=2$.

(2в) Заметим, что условия (5a) $-(5$ д) дают условия (3a)-(3д). Поэтому $L$ проксиминально по теореме 3 . По теореме 1 для $L$ вьполняются условия (1а) и (1в), следовательно, (2а) и (2в), а также (2). Так как $Q \backslash S_{\lambda} \subset \stackrel{\circ}{N}_{z}$ согласно (2) и (5б), то (2б) следует из (5в).

(2г) Если $I=\left\{t_{1}\right\}$, но $\lambda\left\{t_{1}\right\}=0 \forall \lambda \in L^{\perp}$, то это противоречит (5б).

Tеорема 6. Пусть бикомпакт $Q$, мера $\nu \in C(Q)^{*}$ и функиия $f \in C(Q, \widetilde{\mathbb{C}})$ таковbl, чmo

(6a) $S_{\nu}=Q$

(6б) $d \nu / d|\nu| \in C(Q)$;

(6в) $\forall z \in \mathbb{C} \quad N_{z, f}=I_{z} \cup B_{z}$, где $I_{z}=I \cap N_{z, f}$ пусто или состоит из одной точки, $a|\nu| B_{z}=0$ (это әквивалентно условиям (5в) и (5г));

(6г) для любого $z \in \mathbb{C}$ функция $(f(t)-z) /|f(t)-z|$, определенная в точках $t \in Q \backslash N_{z, f}$, продолжается до функиии $\psi_{z} \in C(Q)$;

(6д) $\int_{Q}|f(t)|^{n-1} d|\nu|<\infty$.

Тогда если

$$
\mu_{i} e=\int_{e} f(t)^{i-1} d \nu \quad \forall e \in \Sigma, \quad \forall i=1, \ldots, n,
$$

то подпространство

$$
L=\left\{\mu_{1}, \mu_{2}, \ldots, \mu_{n}\right\}_{\perp}=\left\{x \in C(Q): \int_{Q} x d \mu_{i}=0 \forall i=1, \ldots, n\right\}
$$

является чебышевским.

ДокАЗАТЕЛЬСТво. Заметим вначале, что из

$$
\int_{Q}|f(t)|^{n-1} d|\nu|<\infty
$$

следует

$$
\int_{Q}|f(t)|^{i-1} d|\nu|<\infty \quad \text { при } i=2,3, \ldots, n-1,
$$

так что $\mu_{i} \in C(Q)^{*}$. Проверим условия теоремы 2 .

(2а) Пусть $\lambda=\sum_{1}^{r+1} \alpha_{i} \mu_{i}$, т.е.

$$
\lambda e=\int_{e}^{r+1} \sum_{1} \alpha_{i} f(t)^{i-1} d \nu=\int_{e} p[f(t)] d \nu,
$$

где $p$ - алгебраический полином степени $r<n$. Как и в лемме 8, получим

$$
g(t) \frac{d \lambda}{d|\lambda|}(t)=g(t) \frac{p[f(t)]}{|p[f(t)]|}=g(t) \prod_{1}^{r} \frac{f(t)-z_{i}}{\left|f(t)-z_{i}\right|} \quad \lambda \text {-почти всюду на } \bigcap_{1}^{r}\left(Q \backslash N_{z_{i}}\right),
$$


где $z_{i}-$ корни полинома $p$ в $\mathbb{C} ;$ можно считать, что $\alpha_{r+1}=1$. В силу $(6 б)$ и $(6 г)$

$$
\frac{d \lambda}{d|\lambda|}=g \prod_{1}^{r} \psi_{z_{i}} \in C(Q)
$$

(2б) Поскольку $Q \backslash S_{\lambda}=G$ открыто в $Q$ и

$$
\int_{G}|p[f(t)]| d|\nu|=|\lambda| G=0
$$

то $p[f(t)]=0 \quad \nu$-почти всюду на $G$, а по непрерывности $\forall t \in G$. Но $p(z)=0$ в $r \leqslant n-1$ точках $z_{1}, \ldots, z_{r} \in \mathbb{C}$, а $N_{z_{i}}=I_{z_{i}} \cup B_{z_{i}}$. Тогда $G \subset \bigcup_{1}^{r} N_{z_{i}}=I^{\prime} \cup B$, где $I^{\prime}=\bigcup_{1}^{r} I_{z_{i}}$, $B=\bigcup_{1}^{r} B_{z_{i}}$. Поскольку открытое $G \backslash I^{\prime} \subset B$, то $B=\varnothing$ в силу (6а) и (6в), а $G \subset I^{\prime}$, значит, $\left|Q \backslash S_{\lambda}\right| \leqslant n-1$.

(2в) Так как

$$
|\lambda| e=\int_{e}|p[f(t)]| d|\nu|
$$

то для $E=Q \backslash \bigcup_{1}^{r} I_{z_{i}}$ имеем

$$
\int_{e} \frac{d|\lambda|}{|p[f(t)]|}=\int_{e} \frac{|p[f(t)]| d|\nu|}{|p[f(t)]|}=|\nu| e \quad \forall e \subset E, \quad e \in \Sigma .
$$

Тогда из $e \subset Q^{\prime},|\lambda| e=0, e \subset E$ следует $|\nu| e=0$. Если же $e \subset \cup N_{z_{i}}$, то $e \subset \cup B_{z_{i}}$, и $|\nu| e=0$ согласно (6в). Таким образом, $\nu \ll \lambda$ на $Q^{\prime}$. Если $\lambda^{\prime}=\sum \alpha_{i}^{\prime} \mu_{i}$, то

$$
\left|\lambda^{\prime}\right| e=\int_{e}\left|\sum \alpha_{i}^{\prime} f(t)^{i-1}\right| d|\nu|=0
$$

Поэтому и $\lambda^{\prime} \ll \lambda$ на $Q^{\prime}$.

$(2 г)$ Если $I=\left\{t_{1}, \ldots, t_{q}\right\}$ и $q<n$, то определитель Вандермонда

$$
\operatorname{det}\left\|\mu_{i}\left\{t_{k}\right\}\right\|_{k \leqslant q}^{i \leqslant q}=\operatorname{det}\left\|f\left(t_{k}\right)^{i-1} \nu\left\{t_{k}\right\}\right\|=\prod_{l=1}^{q} \nu\left\{t_{l}\right\} \prod_{j<i \leqslant q}\left[f\left(t_{i}\right)-f\left(t_{j}\right)\right] \neq 0
$$

в силу $(6 \mathrm{a})$ и (6в). Поэтому (2г) вьполняется.

Теорема 7. Пусть $n \geqslant 2$ и $Q$ таковы, что в вещественном $C(Q)$ существуют чебышевские подпространства коразмерности п. Тогда в комплексном $C(Q)$ существуют чебишевские подпространства любой конечной коразмерности.

ДокАЗАТЕЛьСтво. По теореме Брауна [4, теорема 2] существуют мера $\nu \in C(Q, \mathbb{R})$ * и функция $f \in C(Q, \mathbb{R})$ такие, что $S_{\nu}=Q, \nu \geqslant 0$, условие (6в) выполняется $\forall z \in \mathbb{R}$ и каждая точка $t \in Q$ - локальньй экстремум функции $f$ (это эквивалентно условию (6г) в вешественном случае). Рассмотрим меры $\mu_{i}$ из теоремы 6 . Это вешественные меры, но подпространство $L$ комплексное. Легко видеть, что вьполняются условия теоремы 6 . 
Теорема 8. Пусть $n \geqslant 2, Q$ - бикомпакт. Для того чтобы в комплексном $\mathrm{C}(Q)$ существовало чебышевское подпространство коразмерности $n$ достаточно, а если $Q$ метризуемо, то и необходимо, чтобь $Q=\bar{I},|I| \leqslant \mathfrak{N}_{0}$.

В вещественном случае теорема установлена Гаркави [2]. Мы используем некоторые идеи его доказательства.

ДокАЗАТЕЛЬСтво. Достаточность. Можно применить теорему 7 и результат Гаркави или положить

$$
\nu\left\{t_{i}\right\}=2^{-i}, \quad|\nu| Q^{\prime}=0, \quad f\left(t_{i}\right)=i, \quad f(t)=\infty \quad \forall t \in Q^{\prime}
$$

где $\left\{t_{1}, t_{2}, \ldots, t_{n}, \ldots\right\}=I$, и применить теорему 6 .

Необходимость. Пусть $d$ - метрика в $Q, L$ - чебышевское подпространство в $C(Q)$, $\operatorname{codim} L=n \geqslant 2$. Тогда $L$ проксиминально, а по следствию 1

$$
\bigcup_{\lambda \in L^{\perp}} S_{\lambda}=Q .
$$

Отсюда и из леммы 2 или из сепарабельности $Q$ следует, что $|I| \leqslant \mathfrak{N}_{0}$. По теореме 4 для некоторых мер $\mu, \nu \in L^{\perp} \backslash\{0\}$ выполняются условия $(3 \mathrm{a})-(3 д), S_{\nu}=Q$ и $f=d \mu / d \nu \in C(Q, \widetilde{\mathbb{C}})$ отлична от константы. Положим

$$
\begin{gathered}
v_{t}(u)=\psi_{z}(u), \quad \text { где } t, u \in Q^{\prime}, \quad z=f(t) \quad\left(\text { см. (3д)); } \quad c_{j}=\exp \left(i \frac{2 \pi j}{3}\right) ;\right. \\
V_{n} t=\left\{u \in Q^{\prime}: d(t, u)<\frac{1}{n}\right\}, \quad V_{n}^{\prime} t=\left\{u \in V_{n} t: f(u) \neq f(t)\right\} \\
A_{j}^{n}=\left\{t \in Q^{\prime}:\left|v_{t}(u)-c_{j}\right|<\sqrt{2} \forall u \in V_{n}^{\prime} t\right\}, \quad j=1,2,3, \quad n \in \mathbb{N} .
\end{gathered}
$$

Пусть $t \in Q^{\prime}$, тогда $v_{t}(u) \rightarrow v_{t}(t)$ при $u \rightarrow t$. Поскольку $\left|v_{t}(t)\right|=1$, сушествует $j \in\{1,2,3\}$ с $\left|v_{t}(t)-c_{j}\right|<\sqrt{2}$. Сушествует, далее, $n \in \mathbb{N}$ с $\left|v_{t}(u)-c_{j}\right|<\sqrt{2} \forall u \in V_{n}^{\prime} t$. Это означает, что

$$
t \in A_{j}^{n}, \quad Q^{\prime}=\bigcup_{j, n} A_{j}^{n} .
$$

Допустим, что найдутся $u, t \in A_{j}^{n}$ с $d(u, t)<1 / n, f(u) \neq f(t)$. Тогда $\left|v_{t}(u)-c_{j}\right|<\sqrt{2}$ и $\left|v_{u}(t)-c_{j}\right|<\sqrt{2}$ по определению $A_{j}^{n}$. Но это невозможно, так как $v_{t}(u)=-v_{u}(t)$ (при $\left.f(u) \neq f(t)\left|v_{t}(u)\right|=\left|v_{u}(t)\right|=1\right)$. Значит, если $t \in A_{j}^{n}$, то $A_{j}^{n} \cap V_{n}^{\prime} t=\varnothing$, тогда $f(u)=f(t) \forall u \in V_{n} t$. $A_{j}^{n}$ имеет конечную $\varepsilon$-сеть при $\varepsilon=1 / n$, следовательно, $f\left(A_{j}^{n}\right)$ конечно, $f(Q)=f\left(Q^{\prime}\right) \cup f(I)$ счетно, $f(Q)=\left\{z_{1}, z_{2}, \ldots\right\}$. Тогда $Q=\cup N_{z_{i}}$. По лемме 7 $\stackrel{\circ}{N}_{z_{i}}=Q \backslash S_{\lambda}\left(\lambda=\mu-z_{i} \nu\right)$. По теореме $2\left|\stackrel{\circ}{N}_{z_{i}}\right|<n$. Далее, $Q=\left(\cup \stackrel{\circ}{N}_{z_{i}}\right) \cup\left(\cup \partial N_{z_{i}}\right)=I^{\prime} \cup B$. $B=Q \backslash I^{\prime}$ замкнуто и $|\nu| B=0$, следовательно, $B$ нигде не плотно, а тогда $Q=\bar{I}^{\prime}$, что ввиду $I^{\prime} \subset I$ заканчивает доказательство теоремы 8. 
Теорема 9. Пусть бикомпакт $Q$ имеет следующие свойства:

(9а) замыкания непересекающихся открытых $F_{\sigma}$-множеств в $Q$ не пересекаются;

(9б) существует мера $\nu \in C(Q)^{*}$ с $S_{\nu}=Q$;

(9в) существует функиия $f \in C(Q)$ такая, что $\forall z \in \mathbb{C}\left|\stackrel{\circ}{N}_{z, f}\right| \leqslant 1, a|\nu|\left(\partial N_{z, f}\right)=0$.

Тогда в $C(Q)$ существуют чебышевские подпространства любой конечной коразмерности.

В вешественном случае аналогичное утверждение для экстремально несвязных бикомпактов, которые удовлетворяют условию (9a), имеется у Гаркави [3, теорема 5$]$.

ДоКАЗАТЕЛЬСТво ТЕОРЕмЫ 9. Проверим условия (6б) и (6г) (остальные очевидны).

(6б) Если указанная в (9б) мера не удовлетворяет условию (6б), то можно взять $\nu^{\prime}=|\nu|$, тогда $d \nu^{\prime} / d\left|\nu^{\prime}\right| \equiv 1$.

$(6 г)$ Допустим, что (6г) не вьполняется, тогда существуют $z \in \mathbb{C}$ и направленности $t_{\alpha}, t_{\beta} \rightarrow t_{0}$ такие, что для $\varphi(t)=f(t)-z$ имеем

$$
\frac{\varphi\left(t_{\alpha}\right)}{\left|\varphi\left(t_{\alpha}\right)\right|} \rightarrow w_{1}, \quad \frac{\varphi\left(t_{\beta}\right)}{\left|\varphi\left(t_{\beta}\right)\right|} \rightarrow w_{2} \neq w_{1}
$$

Рассмотрим в $\mathbb{C}$ открытые множества

$$
G_{j}=\left\{z \in \mathbb{C}:\left|\frac{z}{|z|}-w_{j}\right|<\left|\frac{w_{1}-w_{2}}{2}\right|\right\}, \quad j=1,2 .
$$

Тогда $\varphi^{-1}\left(G_{1}\right), \varphi^{-1}\left(G_{2}\right)$ - открытые в $Q$ непересекаюшиеся $F_{\sigma}$-множества. Их замыкания, очевидно, содержат точку $t_{0}$, что противоречит (9б).

\section{СПИСОК ЦИТИРОВАННОЙ ЛИТЕРАТУРЫ}

[1] Phelps R. R. Čebyšev subspaces of finite codimension in $C(X)$ // Pacific J. Math. 1963. V. 13. №2. P. $647-655$.

[2] Гаркави А. Л. Задача Хелли и наилучшее приближение в пространстве непрерывных функций // Изв. АН СССР. Сер. матем. 1967. Т. 31. № 3. С. 641-656.

[3] Гаркави А. Л. О компактах, допускающих чебышевские системы мер // Матем. сб. 1967. T. 74 (116). №2. C. 209-217.

[4] Brown A. L. Chebyshev subspaces of finite codimension in spaces of continuous functions // J. Austral. Math. Soc. Ser. A. 1978. V. 26. № 1. P. 99-109.

[5] Власов Л. П. Существование элементов наилучшего приближения в комплексном $C(Q) / /$ Матем. заметки. 1986. Т. 40. №5. С. 627-634.

[6] Данфорд Н., Шварц Дж. Линейные операторы. Общая теория. М.: ИЛ, 1962.

[7] Singer I. Best approximation in normed linear spaces by elements of linear subspaces. Berlin: Springer Verlag, 1970.

[8] Власов Л. П. Аппроксимативные свойства подпространств конечной коразмерности в $C(Q)$ // Матем. заметки. 1980. Т. 28. №2. С. 205-222.

Институт математики и механики УрО РАН,

г. Екатеринбург 